PROCEEDINGS OF THE

AMERICAN MATHEMATICAL SOCIETY

Volume 131, Number 7, Pages 1999-2006

S 0002-9939(03)06955-7

Article electronically published on February 5, 2003

\title{
LIMITS OF RESIDUALLY IRREDUCIBLE $p$-ADIC GALOIS REPRESENTATIONS
}

\author{
CHANDRASHEKHAR KHARE
}

(Communicated by David E. Rohrlich)

\begin{abstract}
In this paper we produce examples of converging sequences of Galois representations, and study some of their properties.
\end{abstract}

\section{INTRODUCTION}

Consider a continuous representation

$$
\rho: G_{L} \rightarrow G L_{m}(K)
$$

of the absolute Galois group $G_{L}$ of a number field $L$, with $K$ a finite extension of $\mathbf{Q}_{p}$, with $\mathcal{O}$ its ring of integers, | | its norm, and $k$ its residue field. Then $\rho$ has an integral model taking values in $G L_{m}(\mathcal{O})$, and the semisimplification of its reduction modulo the maximal ideal $\mathrm{m}$ of $\mathcal{O}$, denoted by $\bar{\rho}$, is independent of the choice of integral model. We assume that $\bar{\rho}$ is absolutely irreducible and, in fact, we assume that all the $p$-adic representations considered in this paper are residually absolutely irreducible.

Definition 1. An infinite sequence of (residually absolutely irreducible) continuous representations $\rho_{i}: G_{L} \rightarrow G L_{m}(K)$ tends to $\rho: G_{L} \rightarrow G L_{m}(K)$, if $\mid \operatorname{tr}\left(\rho_{i}(g)\right)-$ $\operatorname{tr}(\rho(g)) \mid \rightarrow 0$ uniformly for all $g \in G_{L}$. We also say that the $\rho_{i}$ 's converge to $\rho$, or $\rho$ is their limit point.

By Theorem 1 of [Ca], which we can apply because of our blanket assumption of residual absolute irreducibility, this is equivalent to saying that given any integer $n$, for all $i>>0$, the reduction mod $\mathrm{m}^{n}, \rho_{i, n}$, of (an integral model of) $\rho_{i}$ is isomorphic to the reduction mod $\mathrm{m}^{n}, \rho_{n}$, of (an integral model of) $\rho$. Note that we are not assuming that the $\rho_{i}$ 's (or $\rho$ ) are finitely ramified, though we do know by the main theorem of [KhRa] that the density of primes which ramify in a given $\rho_{i}$ is 0 .

In this paper we study the limiting behavior of the lifts produced in [R1] and completely characterize the limit points of these lifts (see Theorem 1 below). This suggests another approach to certain special cases of the modularity lifting theorems of Wiles, Taylor-Wiles, et al. In the process we construct many sequences of converging $p$-adic Galois representations (of fixed determinant and fixed ramification behaviour at $p$ ). This raises many questions that can be posed far more easily than answered.

Received by the editors February 5, 2002.

2000 Mathematics Subject Classification. Primary 11R32, 11R39.

(C)2003 American Mathematical Society 
Consider $\bar{\rho}: G_{\mathbf{Q}} \rightarrow G L_{2}(k)$ that satisfies the conditions of [R1], namely:

- $\bar{\rho}$ and $\operatorname{Ad}^{0}(\bar{\rho})$ are absolutely irreducible Galois representations, and the finite field $k$ of characteristic $p$ is the minimal field of definition of $\bar{\rho}$.

- The (prime to $p$ ) Artin conductor $N(\bar{\rho})$ of $\bar{\rho}$ is minimal amongst its twists. Denote by $S$ the set of primes given by the union of the places where $\bar{\rho}$ is ramified and $\{p, \infty\}$.

- If $\bar{\rho}$ is even, then for the decomposition group $G_{p}$ above $p$ we assume that $\left.\bar{\rho}\right|_{G_{p}}$ is not twist equivalent to $\left(\begin{array}{ll}\chi & 0 \\ 0 & 1\end{array}\right)$ or twist equivalent to the indecomposable representation $\left(\begin{array}{cc}\chi^{p-2} & * \\ 0 & 1\end{array}\right)$ where $\chi$ is the $\bmod p$ cyclotomic character.

- If $\bar{\rho}$ is odd, we assume $\left.\bar{\rho}\right|_{G_{p}}$ is not twist equivalent to the trivial represen-

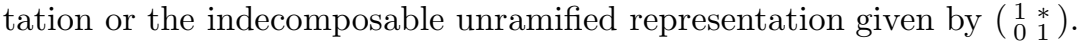

- $p \geq 7$ and the order of the projective image of $\bar{\rho}$ is a multiple of $p$.

Let $Q=\left\{q_{1}, \ldots, q_{n}\right\}$ be a finite set of primes such that $q_{i} \neq \pm 1 \bmod p$, unramified in $\bar{\rho}$, and the ratio of the eigenvalues of $\bar{\rho}\left(\mathrm{Frob}_{q_{i}}\right)$ equal to $q_{i}^{ \pm 1}$. We will call the primes as in $Q$ above Ramakrishna primes for $\bar{\rho}$ or $R$-primes for short (suppressing the $\bar{\rho}$ which is fixed). We consider the deformation ring $R_{S \cup Q}^{Q-n e w}$ of [KR] (see Definition 1 of loc. cit.). To orient the reader we recall the definition of $R_{S \cup Q}^{Q-n e w}$. For this we need:

Definition 2. If $q$ is a prime, $G_{\mathbf{Q}_{q}}$ the absolute Galois group of $\mathbf{Q}_{q}$ and $R$ a complete Noetherian local ring with residue field $k$, a continuous representation $\rho: G_{\mathbf{Q}_{q}} \rightarrow G L_{2}(R)$ is said to be special if up to conjugacy it is of the form $\left(\begin{array}{cc}\varepsilon \chi^{\prime} & * \\ 0 & \chi^{\prime}\end{array}\right)$ for $\varepsilon$ the $p$-adic cyclotomic character, and $\chi^{\prime}: G_{\mathbf{Q}_{q}} \rightarrow R^{*}$ a continuous character. A continuous representation $\tilde{\rho}: G_{\mathbf{Q}} \rightarrow G L_{2}(R)$, is said to be special at a prime $q$ if $\left.\tilde{\rho}\right|_{D_{q}}$, with $D_{q}$ a decomposition group at $q$, is special.

Then $R_{S \cup Q}^{Q-n e w}$ is the universal ring that parametrizes deformations of $\bar{\rho}$ that are minimally ramified at $S$ (in the sense of [R1]: thus when $\bar{\rho}$ is even there is no condition at $p$ ) and such that at primes $q \in Q$ these deformations are special. The ring $R_{S \cup Q}^{Q-n e w}$ is a complete Noetherian local $W(k)$-algebra, with $W(k)$ the Witt vectors of $k$. The deformation rings considered here are for the deformation problem with a certain fixed (arithmetic) determinant character, and all the deformations of $\bar{\rho}$ we consider will have this fixed determinant character.

Definition 3. A finite set of $R$-primes $Q$ is said to be auxiliary if $R_{S \cup Q}^{Q-n e w} \simeq W(k)$.

In [R1] auxiliary sets $Q$ of the above type were proven to exist. The representation corresponding to $R_{S \cup Q}^{Q-n e w} \simeq W(k)$ is denoted by $\rho_{S \cup Q}^{Q-n e w}$. We will call these lifts Ramakrishna lifts of $\bar{\rho}$ or $R$-lifts for short (suppressing the $\bar{\rho}$ which is fixed).

Theorem 1. A continuous representation $\rho: G_{\mathbf{Q}} \rightarrow G L_{2}(W(k))$ that is a deformation of $\bar{\rho}$, is a limit point of distinct R-lifts, if and only if $\rho$ is unramified outside $S$ and the set of all $R$-primes, and minimally ramified at primes of $S$.

Remark. Thus, we have a complete description of the " $p$-adic closure" of $R$-lifts. Note that, in particular, each $R$-lift is a limit point of other $R$-lifts. Note also that any deformation $G_{\mathbf{Q}} \rightarrow G L_{2}(K)$ of $\bar{\rho}$ that is a limit point of $R$-lifts has a model that takes values in $G L_{2}(W(k))$. The above theorem can be viewed in a sense as producing an "infinite fern" structure (in the sense of Mazur) in the set of all $R$-lifts 


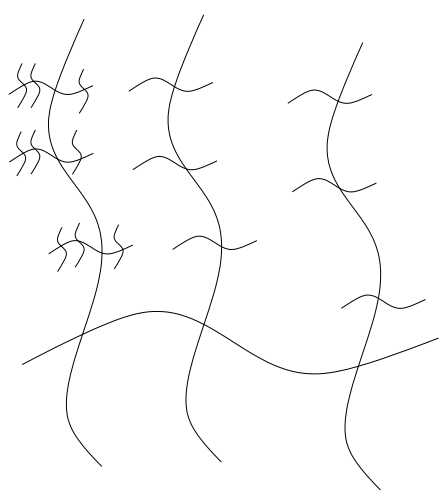

Figure 1. An infinite fern

of a given $\bar{\rho}$ as above (see the picture below). From the proof of Theorem 1 above, we in fact can deduce that each $R$-lift gives rise to infinitely many "splines" passing through it, where a "spline" consists of a sequence of $R$-lifts converging to it, and each element in a spline gives rise to its own infinitely many splines. Missing from the picture are the limit points of $R$-lifts which themselves are not $R$-lifts and which the theorem above characterizes completely.

In Section 2 we prove Theorem 1 which is a simple consequence of the methods of [R1] and [T1]. In Section 3 we prove a result about converging sequences of representations arising from newforms, and point out a possible approach to the lifting theorems of Wiles, et al. that is suggested by the work here. In Section 4 we raise questions about rationality and motivic properties of converging sequences of $p$-adic Galois representations.

\section{Converging sequences of Galois representations}

We now prove Theorem 1 which follows from the methods of [R1] and [T1. For the proof we need the following lemma which follows from the methods of [R1] (see also Lemma 1.2 of [T1]) and Lemma 8 of [KR].

Lemma 1. Let $\rho_{n}: G_{\mathbf{Q}} \rightarrow G L_{2}\left(W(k) /\left(p^{n}\right)\right)$ be a lift of $\bar{\rho}$ that is unramified outside $S$ and the set of all $R$-primes, minimally ramified at primes of $S$, and special at all the primes outside $S$ at which it is ramified. Let $Q_{n}^{\prime}$ be any finite set of primes that includes the primes of ramification of $\rho_{n}$ such that $Q_{n}^{\prime} \backslash S$ contains only $R$-primes and such that $\left.\rho_{n}\right|_{D_{q}}$ is special for $q \in Q_{n}^{\prime} \backslash S$. Then there exists a finite set of primes $Q_{n}$ that contains $Q_{n}^{\prime}$, such that $\left.\rho_{n}\right|_{D_{q}}$ is special for $q \in Q_{n} \backslash S, Q_{n} \backslash S$ contains only $R$-primes and $Q_{n} \backslash S$ is auxiliary.

Proof. We use [R1] and Lemma 8 of [KR] to construct an auxiliary set of primes $T_{n}$ such that $\left.\rho_{n}\right|_{D_{q}}$ is special for $q \in T_{n}$. Then as $Q_{n}^{\prime} \backslash S$ contains only $R$-primes, it follows (using notation of [R1]) from Proposition 1.6 of [W] that the kernel and cokernel of the map

$$
H^{1}\left(G_{S \cup T_{n} \cup Q_{n}^{\prime}}, \operatorname{Ad}^{0}(\bar{\rho})\right) \rightarrow \bigoplus_{v \in S \cup T_{n} \cup Q_{n}^{\prime}} H^{1}\left(G_{v}, \operatorname{Ad}^{0}(\bar{\rho})\right) / \mathcal{N}_{v}
$$


have the same cardinality. Then using Proposition 10 of [R1], or Lemma 1.2 of [T1], and Lemma 8 of [KR], we can augment the set $S \cup T_{n} \cup Q_{n}^{\prime}$ to get a set $Q_{n}$ as in the statement of the lemma.

We are now ready to prove Theorem 1 If $\rho: G_{\mathbf{Q}} \rightarrow G L_{2}(W(k))$ is a limit point of $R$-lifts, then it is clear that $\rho$ is unramified outside $S$ and the set of all $R$-primes, and minimally ramified at primes of $S$. We prove the converse. So let $\rho$ satisfy the conditions of Theorem 1 and recall that we denote by $\rho_{n}$ the reduction modulo $p^{n}$ of $\rho$. It is easily checked that if $q$ is an $R$-prime, then any deformation of $\left.\bar{\rho}\right|_{D_{q}}$ to a ramified $p$-adic representation is special; this follows from the structure of tame inertia and the fact that $q^{2} \neq 1 \bmod p$. Furthermore, from the method of proof of Proposition 1 of [KhRa], we easily deduce that the set of primes $q$ for which $\left.\rho\right|_{D_{q}}$ is special is of density 0. Thus using Cebotarev and the assumptions on $\bar{\rho}$ in the introduction, we choose a finite set of primes $Q_{n}^{\prime}$ such that

- $Q_{n}^{\prime} \backslash S$ consists of $R$-primes and $\left.\rho_{n}\right|_{D_{q}}$ is special for $q \in Q_{n}^{\prime} \backslash S$,

- $Q_{n}^{\prime}$ contains all the ramified primes of $\rho_{n}$,

- for some prime $q \in Q_{n}^{\prime} \backslash S,\left.\rho\right|_{D_{q}}$ is not special.

Using Lemma 11 we complete $Q_{n}^{\prime}$ to a set $Q_{n}$ such that $Q_{n} \backslash S$ is auxiliary and $\left.\rho_{n}\right|_{D_{q}}$ is special for $q \in Q_{n} \backslash S$. Then we claim $\rho_{S \cup Q_{n}}^{Q_{n} \backslash S-n e w} \equiv \rho \bmod p^{n}$. The claim is true as there is a unique representation $G_{\mathbf{Q}} \rightarrow G L_{2}\left(W(k) /\left(p^{n}\right)\right.$ ) (with the determinant that we have fixed) that is unramified outside $S \cup Q_{n}$, minimal at $S$ and special at primes of $Q_{n} \backslash S$ (as $R_{S \cup Q_{n}}^{Q_{n} \backslash S-\text { new }} \simeq W(k)$ ). By construction the sets $Q_{n}$ contain at least one prime at which $\rho$ is not special. Thus, we see that we can pick a subsequence of mutually distinct representations $\rho_{i}$ from the $\rho_{S \cup Q_{n}}^{Q_{n} \backslash S-n e w}$, s such that $\rho_{i} \rightarrow \rho$.

Remark. It is of vital importance that $\rho$ is $G L_{2}(W(k))$-valued since, otherwise, we would not be able to invoke the disjointness results that are used in the proof of Lemma 1 (Lemma 8 of [KR]).

Remark. Theorem 1 can be applied in practice to give many examples of converging sequences of $p$-adic representations: for a non-CM elliptic curve $E_{/ \mathbf{Q}}$ for most primes $p$ the $\bmod p$ representation satisfies the conditions given in the introduction, and the corresponding $p$-adic representation is minimally ramified and $G L_{2}\left(\mathbf{Z}_{p}\right)$ valued.

We end this section with a result that refines the main result of [KhRa].

Proposition 1. If $\rho_{i}: G_{L} \rightarrow G L_{m}(K)$ is a sequence of (residually absolutely irreducible) continuous representations that converges to $\rho$, then the set of primes where any of the $\rho_{i}$ 's is ramified (i.e., $\bigcup \operatorname{Ram}\left(\rho_{i}\right)$ where $\operatorname{Ram}\left(\rho_{i}\right)$ is the set of primes at which $\rho_{i}$ is ramified) is of density zero.

Proof. Denote by $\rho_{i, n}$ (resp., $\rho_{n}$ ) the reduction mod $\mathrm{m}^{n}$ of an integral model of $\rho_{i}$ (resp., $\rho$ ). The proof consists of applying Theorem 1 of [KhRa] twice; more precisely, first its statement, and then its proof. By an application of its statement we conclude that the density of $\bigcup_{i=1}^{n} \operatorname{Ram}\left(\rho_{i}\right)$ is 0 for any $n$. Now applying the proof of Theorem 1 of [KhRa], we define $c_{\rho, n}$ to be the upper density of the set $S_{\rho, n}$ of primes $q$ of $L$ that

- lie above primes which split in $L / \mathbf{Q}$,

- are unramified in $\rho_{1}$ and $\neq p$, 
- $\left.\rho_{n}\right|_{D_{q}}$ is unramified, but there exists a "lift" of $\left.\rho_{n}\right|_{D_{q}}$, with $D_{q}$ the decomposition group at $q$, to a representation $\tilde{\rho}_{q}$ of $D_{q}$ to $G L_{m}(K)$ that is ramified at $q$; by a lift we mean some conjugate of $\tilde{\rho}_{q}$ reduces mod $\mathrm{m}^{n}$ to $\left.\rho_{n}\right|_{D_{q}}$.

We have from KhRa] (see Proposition 1 of loc. cit. which was stated in greater generality than needed there with the present application in mind):

Lemma 2. Given any $\varepsilon>0$, there is an integer $N_{\varepsilon}$ such that $c_{\rho, n}<\varepsilon$ for $n>N_{\varepsilon}$.

To prove Proposition 1 it is enough to show that given any $\varepsilon>0$, the upper density of the set $\bigcup \operatorname{Ram}\left(\rho_{i}\right)$ is $<\varepsilon$. Since $\bigcup_{i=1}^{n} \operatorname{Ram}\left(\rho_{i}\right)$ has density 0 for (the finite) $n$ that is the supremum of the $i$ 's such that $\rho_{i, N_{\varepsilon}}$ is not isomorphic to $\rho_{N_{\varepsilon}}$, and $\rho_{N_{\varepsilon}}$ is finitely ramified, it follows from the lemma above that the upper density of $\bigcup \operatorname{Ram}\left(\rho_{i}\right)$ is $<\varepsilon$. Hence Proposition 1 .

Remark. One can ask for more refined information about the asymptotics of ramified primes in (limits of) residually absolutely irreducible $p$-adic Galois representations. For instance, in Theorem 1 of [KhRa] one can ask (clued by Theorem 10 of [S1]) if the order of growth of ramified primes can be proved to be bounded by $O\left(x^{1-\frac{1}{2 N}+\epsilon}\right)$, where $N$ is the $p$-adic analytic dimension of $\operatorname{im}(\rho)$, for any $\epsilon>0$. Such quantitative refinements asked for by Serre in an e-mail message to the author are difficult and will require a new idea (that goes beyond $\mathrm{KhRa}$ ) and a strong use of effective versions of the Cebotarev density theorem.

\section{Finite And infinite RAmification}

Let $L$ be a number field and $K$ a finite extension of $\mathbf{Q}_{p}$ as before.

Definition 4. We say that a residually absolutely irreducible continuous representation $\rho: G_{L} \rightarrow G L_{n}(K)$ is motivic if $\rho$ arises as a subquotient of the $i^{\text {th }}$ étale cohomology $H^{i}\left(X \times{ }_{L} \bar{L}, K\right)$ of a smooth projective variety $X$ defined over a number field $L$.

A motivic representation is finitely ramified. In $[\underline{\mathrm{R}}$ examples of residually irreducible representations $\rho: G_{\mathbf{Q}} \rightarrow G L_{2}(K)$ were constructed that were infinitely ramified (see also the last section of $[\underline{K R}]$ ). Infinitely ramified $p$-adic representations cannot be motivic. But they can arise as limits of $p$-adic representations that are motivic. Fix an embedding $\overline{\mathbf{Q}} \rightarrow \overline{\mathbf{Q}_{p}}$. Then as in $[\mathbf{R}$. (and the last section of [KR]), there is a sequence of eigenforms $f_{i} \in S_{2}\left(\Gamma_{0}\left(N_{i}\right)\right)$, for a sequence of squarefree integers $N_{i}$ such that $N_{i} \rightarrow \infty$ and $\left(p, N_{i}\right)=1$, new of level $N_{i}$ such that the corresponding $p$-adic representations $\rho_{f_{i}}: G_{\mathbf{Q}} \rightarrow G L_{2}\left(\mathbf{Z}_{p}\right)$ have a $p$-adic limit $\rho$, with $\rho$ infinitely ramified. Such a $\rho$ is non-motivic, but is the limit of motivic $p$-adic representations. Such limits of eigenforms (in the works of Serre and Katz; for instance, cf., $\mathrm{Ka}]$ ) have been considered when varying weights or varying the $p$-power level, while fixing the prime-to- $p$ part of the level.

Proposition 2. Let $f_{i} \in S_{2}\left(\Gamma_{0}\left(N_{i}\right)\right)$ be a sequence of eigenforms with coefficients in a finite extension $K$ of $\mathbf{Q}_{p}$ with $\left(N_{i}, p\right)=1$ and $p \geq 3$, that in the $p$-adic $q$-expansion topology tend to an element $f \in K[[q]]$, such that the corresponding residual representation $\bar{\rho}$ satisfies the conditions in the introduction. The element $f$, that gives rise naturally to a Galois representation $\rho_{f}: G_{\mathbf{Q}} \rightarrow G L_{2}(K)$, is the q-expansion of a classical eigenform (of weight 2) if and only if $\rho_{f}$ is finitely ramified. 
Proof. The only if part is clear. The if part follows from the methods of Wiles (see Chapter 3 of [W] and also [TW]) and their refinements: note that $\rho_{f}$ is finite flat at $p$.

Remark. Applying Theorem 1 when $\bar{\rho}$ is odd and finite flat at $p$, in which case the $R$-lifts are modular by Theorem 1 of $[\mathrm{K}]$, we can construct systematically many examples of sequences of eigenforms $f_{i} \rightarrow f(f \in K[[q]])$, with the levels of $f_{i}$ unbounded and such that $\rho_{f}$ is finitely ramified $(f$, in fact, is then a classical eigenform as above). On the other hand, as recalled above in $[\mathrm{R}$ (see also last section of $[\mathrm{KR}]$ ), we have examples of situations as above with $\rho_{f}$ infinitely ramified.

It will be of interest to see if Proposition 2 could be proved in a more selfcontained manner. The proof above does not use seriously the fact that one does know that $f$ arises as a limit of the classical forms $f_{i}$. If such a proof could be devised, in conjunction with Theorem 1 above and Theorem 1 of $[\mathrm{K}$ (which is due to Ravi Ramakrishna) it would give, in special cases, a simpler approach using $R$ primes to the modularity lifting theorems of Wiles, et al. (see also $[\mathbf{K}]$ ) that directly works with the $p$-adic Galois representation that needs to be proved modular, and if it could be implemented, would avoid (albeit in special cases) the sophisticated deformation theoretic approach of [W].

We elaborate on this: Assume that $\bar{\rho}$ is modular. In Theorem 1 we have characterized the limit points of $R$-lifts. By Theorem 1 of $[\mathrm{K}]$ which proves that the representation corresponding to $R_{S \cup Q}^{Q-n e w} \simeq W(k)$ is modular as a consequence of the isomorphism $R_{S \cup Q}^{Q-n e w} \simeq \mathbf{T}_{S \cup Q}^{Q-\text { new }}$ (using notation of $[\mathrm{K}]$ ), we know that $R$-lifts are modular. Hence, limits of $R$-lifts do arise as limits of $p$-adic representations arising from classical newforms. It only (!) remains to prove that a limit of a converging sequence of $p$-adic representations arising from newforms (say of weight 2 and level prime to $p$ to avoid delicate considerations at $p$ ) that is finitely ramified itself arises from a newform (i.e., prove Proposition 2 without appealing directly to $[\mathrm{W}])$. Note that for a semistable elliptic curve $E$, for all large enough primes $p$ (bigger than 3 for the methods here to directly work unfortunately!), $T_{p}(E)$ is a limit point of $R$-lifts.

Note. In recent work we have indeed been able to give a self-contained approach to a result such as Proposition 2 2 above under some technical restrictions; see [K1].

\section{Questions}

Proposition 2 suggests that a representation that arises as a limit of motivic representations (of "bounded weights"; see Definition [ below) is finitely ramified if and only if it is motivic. We first recall one of the main conjectures in [FM] in a form that is most pertinent for the considerations here.

Conjecture 1 (Fontaine-Mazur). Consider a continuous residually absolutely irreducible representation $\rho: G_{L} \rightarrow G L_{m}(K)$ that is potentially semistable at places above $p$. Then the following are equivalent:

(1) $\rho$ is motivic,

(2) $\rho$ is finitely ramified. 
From our earlier considerations it is natural to ask the following weaker question.

Question 1. Consider a continuous residually absolutely irreducible representation $\rho: G_{L} \rightarrow G L_{m}(K)$ that is potentially semistable at places above $p$ and arises as the limit of motivic representations $\rho_{i}$. Then if $\rho$ is finitely ramified, is $\rho$ motivic?

It seems unlikely that the infinitely ramified representations produced in $[\underline{\mathrm{R}}$ are algebraic (see definition below). This motivates the following considerations.

Definition 5. A continuous (residually absolutely irreducible) representation $\rho$ : $G_{L} \rightarrow G L_{m}(K)$ is said to be algebraic if there is a number field $F$ such that the characteristic polynomial of $\rho\left(\right.$ Frob $\left._{q}\right)$ has coefficients in the ring of integers of $F$ for all primes $q$ which are unramified in $\rho$. The minimal such field is the field of definition of $\rho$.

As by the main theorem of [KhRa], the set of primes at which $\rho$ ramifies is of density 0 , the definition above is a sensible one.

Definition 6. A continuous (residually absolutely irreducible) algebraic representation $\rho: G_{L} \rightarrow G L_{m}(K)$ is said to be of weight $\leq t(t \in \mathbf{Z})$ if for primes $q$ that are unramified in $\rho$, any root $\alpha$ of the characteristic polynomial of $\rho\left(\right.$ Frob $\left._{q}\right)$ satisfies $|\iota(\alpha)| \leq\left|k_{q}\right|^{\frac{t-1}{2}}$ for any embedding $\iota: \overline{\mathbf{Q}} \rightarrow \mathbf{C}$, with $k_{q}$ the residue field at $q$.

Question 2. If $\rho_{i}: G_{L} \rightarrow G L_{m}(K)$ is an infinite sequence of (residually absolutely irreducible) distinct algebraic representations, all of weight $\leq t$ for some fixed integer $t$, converging to $\rho: G_{L} \rightarrow G L_{m}(K)$, and $K_{i}$ the field of definition of $\rho_{i}$, does $\left[K_{i}: \mathbf{Q}\right] \rightarrow \infty$ as $i \rightarrow \infty$ ?

Remark.

- It is observed in $[\overline{\mathrm{R}}]$ (this is a remark of Fred Diamond) that in the situation of Question 2 only finitely many of the $\rho_{i}$ 's can arise from elliptic curves; this is a consequence of the Mordell conjecture which gives that suitable twists of the classical modular curves $X\left(p^{n}\right)$ for $n>>0$ have finitely many $L$-valued points for a given number field $L$.

- If Question 2 has a negative answer, using Proposition 1 we deduce that for a set of primes $\{r\}$ of density one, the characteristic polynomials of $\rho_{i}\left(\right.$ Frob $\left._{r}\right)$ are eventually constant. Hence, we deduce that the characteristic polynomials of $\rho\left(\right.$ Frob $\left._{r}\right)$ are defined and integral over a fixed number field $F$, i.e., $\rho$ is algebraic (in the case when $\rho$ is infinitely ramified this is linked to the questions below).

Question 3. Let $\rho: G_{L} \rightarrow G L_{m}(K)$ be a continuous, residually absolutely irreducible representation that is potentially semistable at places above $p$. Then are the following equivalent:

(1) $\rho$ is motivic,

(2) $\rho$ is finitely ramified,

(3) $\rho$ is algebraic?

In the question above, the equivalence of 1 and 2 is the Fontaine-Mazur conjecture recalled above: the possible equivalence of 3 to 1 and 2 is the main thrust of the question. One might even ask the stronger question: If $\rho: G_{L} \rightarrow G L_{m}(K)$, a continuous, residually absolutely irreducible representation, is algebraic, then is $\rho$ forced to be both finitely ramified and potentially semistable at places above $p$ ? All the questions of this section have a positive answer when $m=1$. 


\section{ACKNOWLEDGEMENTS}

I thank Gebhard Böckle, Christophe Breuil, Dipendra Prasad and Ravi Ramakrishna for helpful correspondence, Kirti Joshi and C. S. Rajan for useful conversations, Haruzo Hida, David Rohrlich, J-P. Serre and the anonymous referee for helpful remarks on the manuscript.

\section{REFERENCES}

[Ca] Carayol, H., Formes modulaires et représentations galoisiennes à valeurs dans un anneau local complet, in p-adic monodromy and the Birch and Swinnerton-Dyer conjecture, 213237, Contemp. Math., 165, AMS, 1994. MR 95i:11059

[FM] Fontaine, J.-M., Mazur, B., Geometric Galois representations, Elliptic curves, modular forms, and Fermat's last theorem, Internat. Press, Cambridge (1995), 41-78. MR 96h:11049

[K] Khare, C., On isomorphisms between deformation rings and Hecke rings, preprint available at http://www.math.utah.edu/ ${ }^{\sim}$ shekhar/papers.html.

[K1] Khare, C., Modularity of p-adic Galois representations via p-adic approximations, in preparation.

[KhRa] Khare, C., Rajan, C. S., The density of ramified primes in semisimple p-adic Galois representations, International Mathematics Research Notices, no. 12 (2001), 601-607. MR 2002e:11066]

[KR] Khare, C., Ramakrishna, R., Finiteness of Selmer groups and deformation rings, preprint available at http://www.math.utah.edu/ shekhar/papers.html.

[Ka] Katz, N., Higher congruences between modular forms, Annals of Math. 101 (1975), 332367.

[R] Ramakrishna, R., Infinitely ramified representations, Annals of Mathematics 151 (2000), 793-815. MR 54:5120

[R1] Ramakrishna, R., Deforming Galois representations and the conjectures of Serre and Fontaine-Mazur, to appear in Annals of Math.

[S1] Serre, J-P., Quelques applications du théorème de densité de Chebotarev, Inst. Hautes Études Sci. Publ. Math., no. 54 (1981), 323-401. MR 83k:12011

[T1] Taylor, R., On icosahedral Artin representations II, preprint.

[TW] Taylor, R., Wiles, A., Ring-theoretic properties of certain Hecke algebras, Ann. of Math. (2) 141 (1995), 553-572. MR 96d:11072

[W] Wiles, A., Modular elliptic curves and Fermat's last theorem, Ann. of Math. 141 (1995), 443-551. MR 96d:11071

Department of Mathematics, University of Utah, 155 S 1400 E, Salt lake City, Utah 84112 - And - School of Mathematics, TIFR, Homi Bhabha Road, Mumbai 400 005, India

E-mail address: shekhar@math.utah.edu

E-mail address: shekhar@math.tifr.res.in 